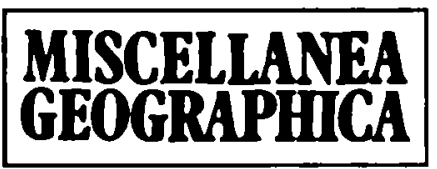

WARSZAWA 1992 Vol. 5

Małgorzata Gutry-Korycka, Urszula Soczyńska

\title{
NUMERICAL SIMULATION OF THE MAN-MADE IMPACT ON HYDROLOGICAL PROCESSES
}

\section{INTRODUCTION}

The paper is devoted to the problem of predicting the hydrological consequences of human activity in physical catchment environment. For their identification and description the physically-based mathematical model relative to the unit basin area (one-dimensional version) has been used. All considerations deal with the active vegetation growth period.

\section{GENERAL ASSUMPTIONS OF THE MATHEMATICAL MODEL}

The concept and algorithm of the model encompass mathematical description of the vertical mass and energy exchange within the air-plant-soil column (Fig. 1). It is composed of the following processes:

- interception and dropping from the plant canopy,

- evapotranspiration,

- soil moisture changes caused by inflitration and evapotranspiration.

\section{INTERCEPTION OF THE PLANT CANOPY}

The model of the interception subsystem is highly flexible in that it allows a description of up to four layers of canopy in differing phytocenoses (GutryKorycka, Soczyńska, 1986). Computational algorithms that consider a dynamic form of the water balance equation for each layer, are based on Fleming's (1975) approach. The model may be used with an arbitrary time interval $\Delta t$. Input to the model is rainfall on the plant canopy $P(t)$ and energy influx (potential evaporation); outputs are: rainfall that reaches the ground surface $P g(t)$ and evapotranspiration from the plant canopy $E(t)$. There are two main parameters: maximum canopy storage $S_{\max }$ and plant density $D$. 
Reading of the constant parameters:

- biometrical: $\quad H_{d} ; D C 1 ; D C 2 ; D C 3 ; D C 4 ; S_{\max 1}, S_{\max 2} ; S_{\max 3} ; S_{\max 4} ; l_{k} ;$ tables $\alpha(\Psi)$

- atmospheric: $\quad k, t ; \alpha ; \alpha_{0} ; \beta ; \beta_{0} ; c_{p 1}=c_{p 2}$

- soil:

$\Theta_{\text {SAT }} ; \mathrm{pF}(\mathrm{I})$ dla I = 1 ... NF; $L ;=K_{s} ;$ Maxg; Ming

- computed: $\quad N N$ - number of steps along the " $z$ " axis: $M$ - number of temporal steps;

$\mathrm{NF}$ - dimension of $\mathrm{pF}$ matrix; $\mathrm{j}$ - number of temporal steps, $\mathrm{j}=1$...M;

$h$ - step along the " $z$ " axis; $i$ - number of spatial steps, $i=1 \ldots N N$;

$\varphi_{1}, \varphi_{2}, \varphi_{3}, \varphi_{4}$ reduction coefficients of evaporation for each plant layer

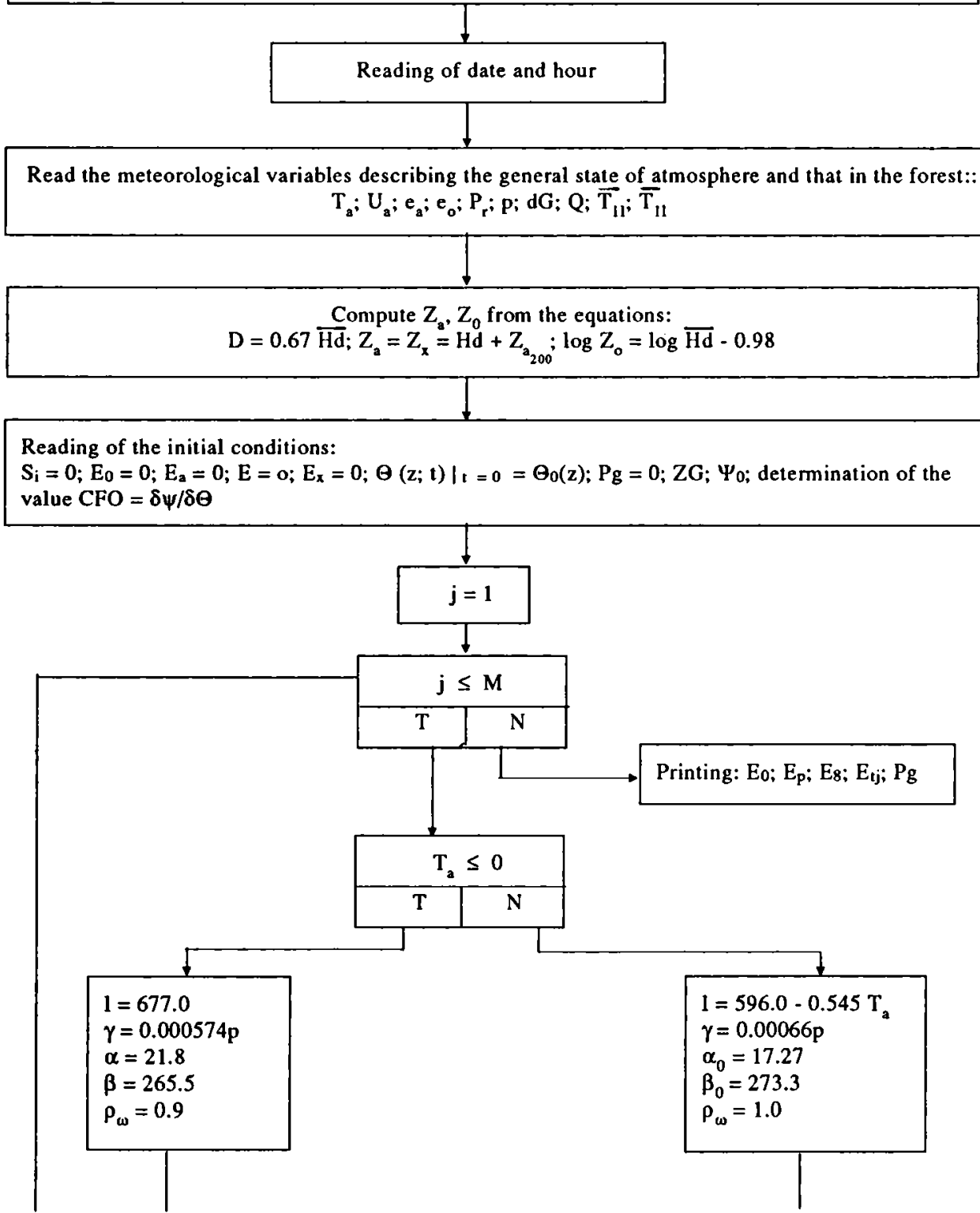




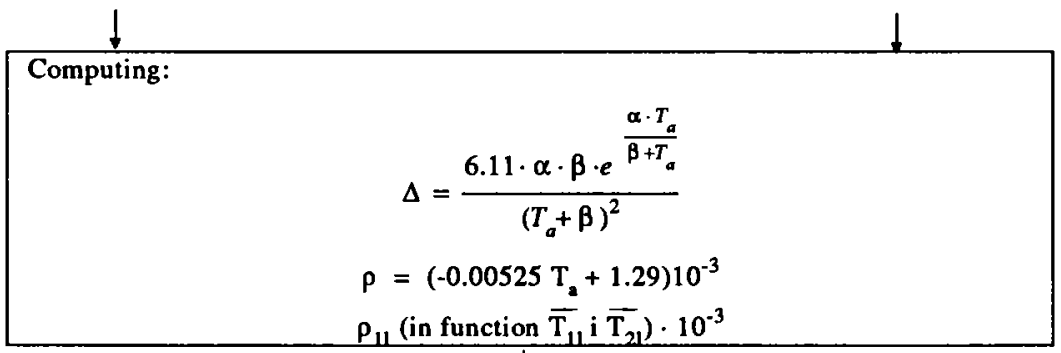

Computing:

$$
\begin{gathered}
d C=\left(\rho_{11} \cdot c_{p 1} \cdot v_{1} \cdot T_{11}-\rho_{21} \cdot c_{p^{2}} \cdot v_{2} \cdot T_{21} / F / d \tau\right. \\
G^{\prime}=d C+d G^{\prime} \\
Q_{L}=a_{4}(+Q) ; Q_{1}=a_{5}(-Q)
\end{gathered}
$$

Computing

$$
E_{p j}=a \frac{\frac{\Delta}{\gamma} \cdot \frac{Q_{L}+G}{l \cdot \rho_{\omega}}-\frac{\rho \cdot L \cdot k^{2} \cdot U_{a}\left(e_{0}-e_{a}\right.}{p \cdot \rho_{w}\left[\ln \left(Z_{o} / Z_{0}\right)^{2}\right]}}{\frac{\Delta}{\gamma}+1}
$$

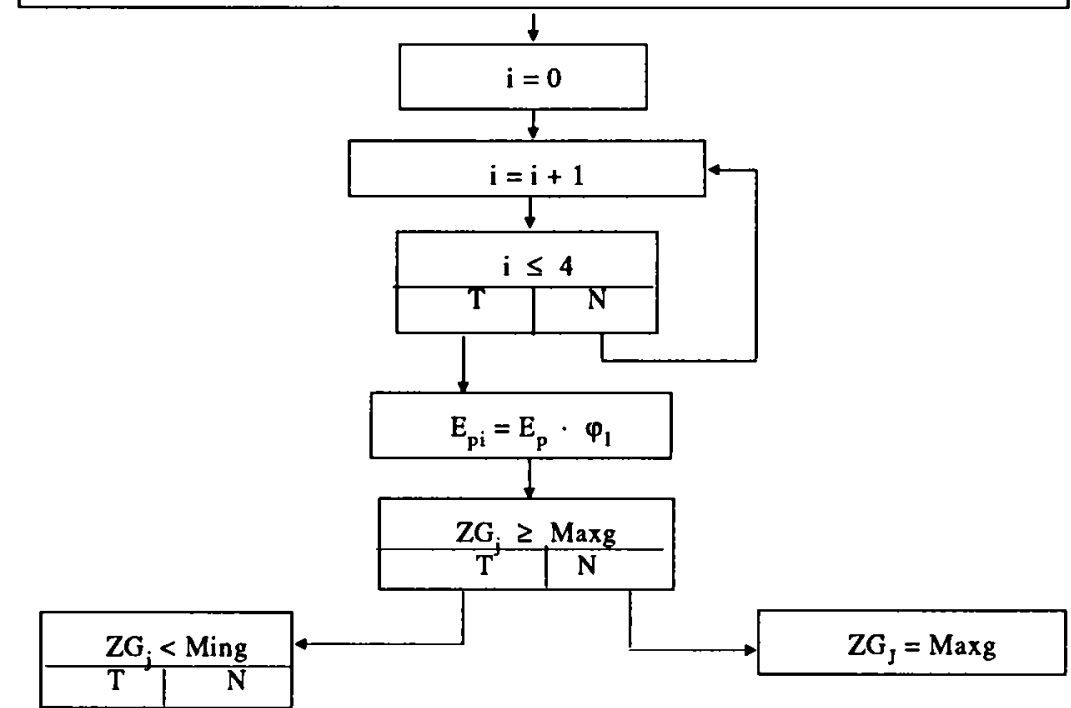

$$
\begin{aligned}
& \text { Computing: (deficit of soil moisture within the layer } 40 \mathrm{~cm}) \\
& \qquad \mathrm{ZGP}=100-\left[\left(M a x g-Z G_{j}\right) \frac{100-40}{\text { Maxg-Ming }}\right]
\end{aligned}
$$




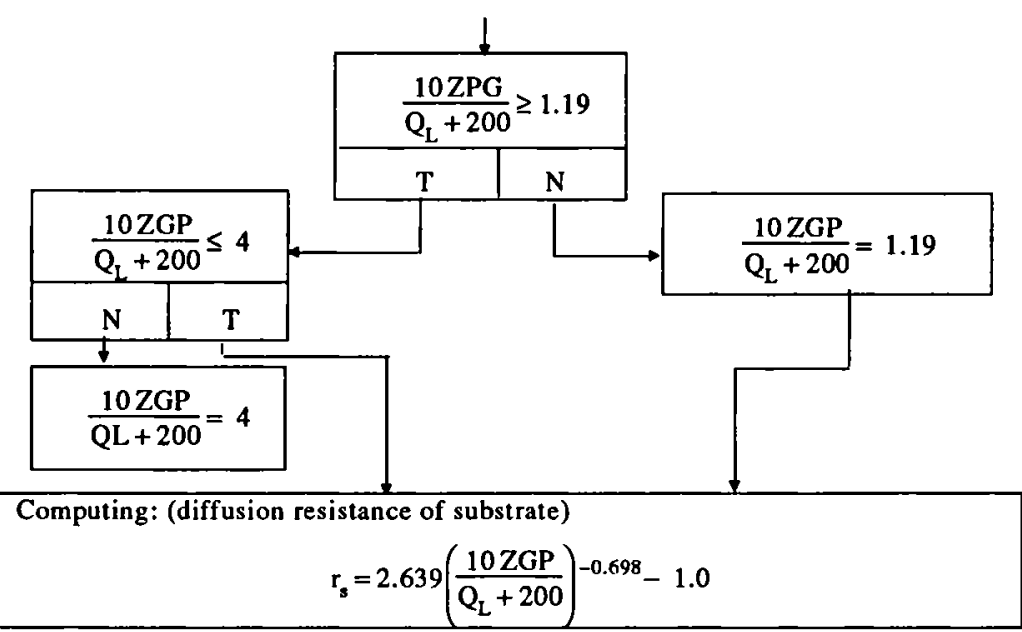

Computing: (actual evapotranspiration)

$$
E_{a j}=E_{p j} \cdot \frac{\frac{\Delta}{\gamma}+1}{\frac{\Delta}{\gamma}+1+r_{s}+U_{a}^{1} k^{2}\left(\ln \frac{z_{a}}{z_{0}}\right)^{2}}
$$

Computing: (evaporation from the forest soil)

$$
E_{s j}=\frac{\Delta}{(\Delta+\gamma) l_{\rho_{w}}} Q_{L}
$$

Computing: (transpiration from the forest)

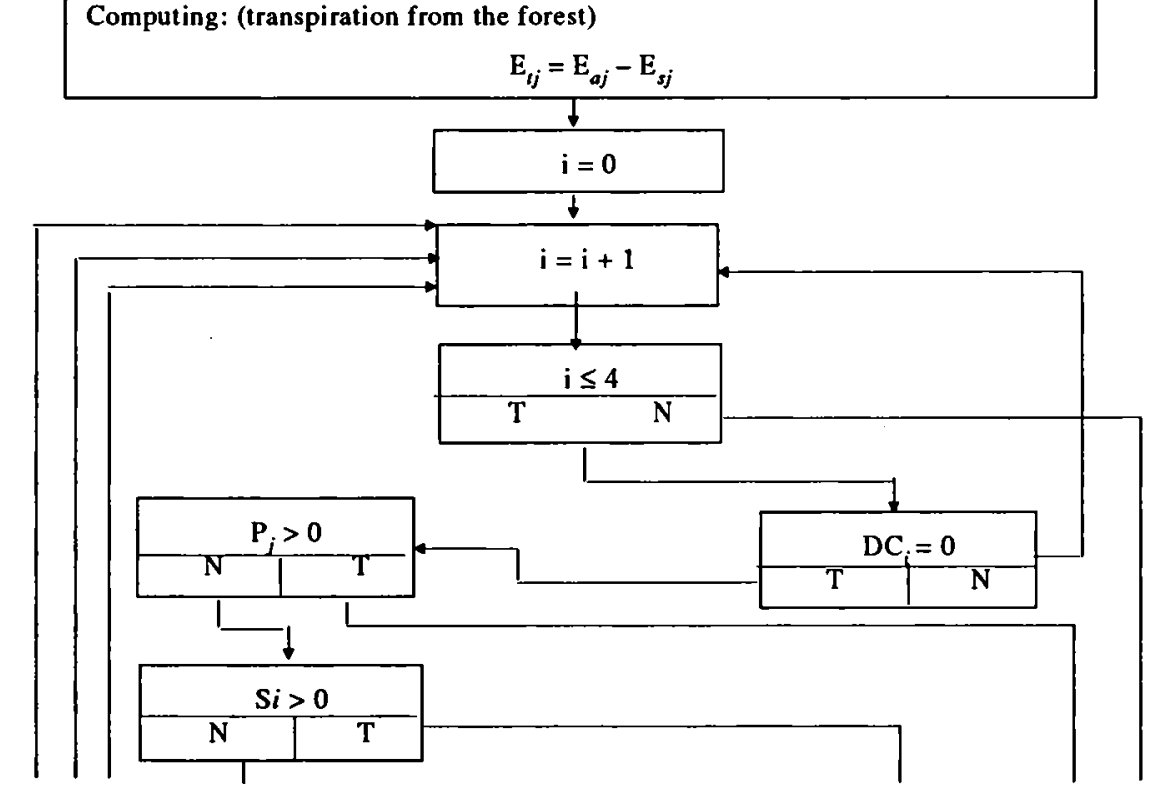




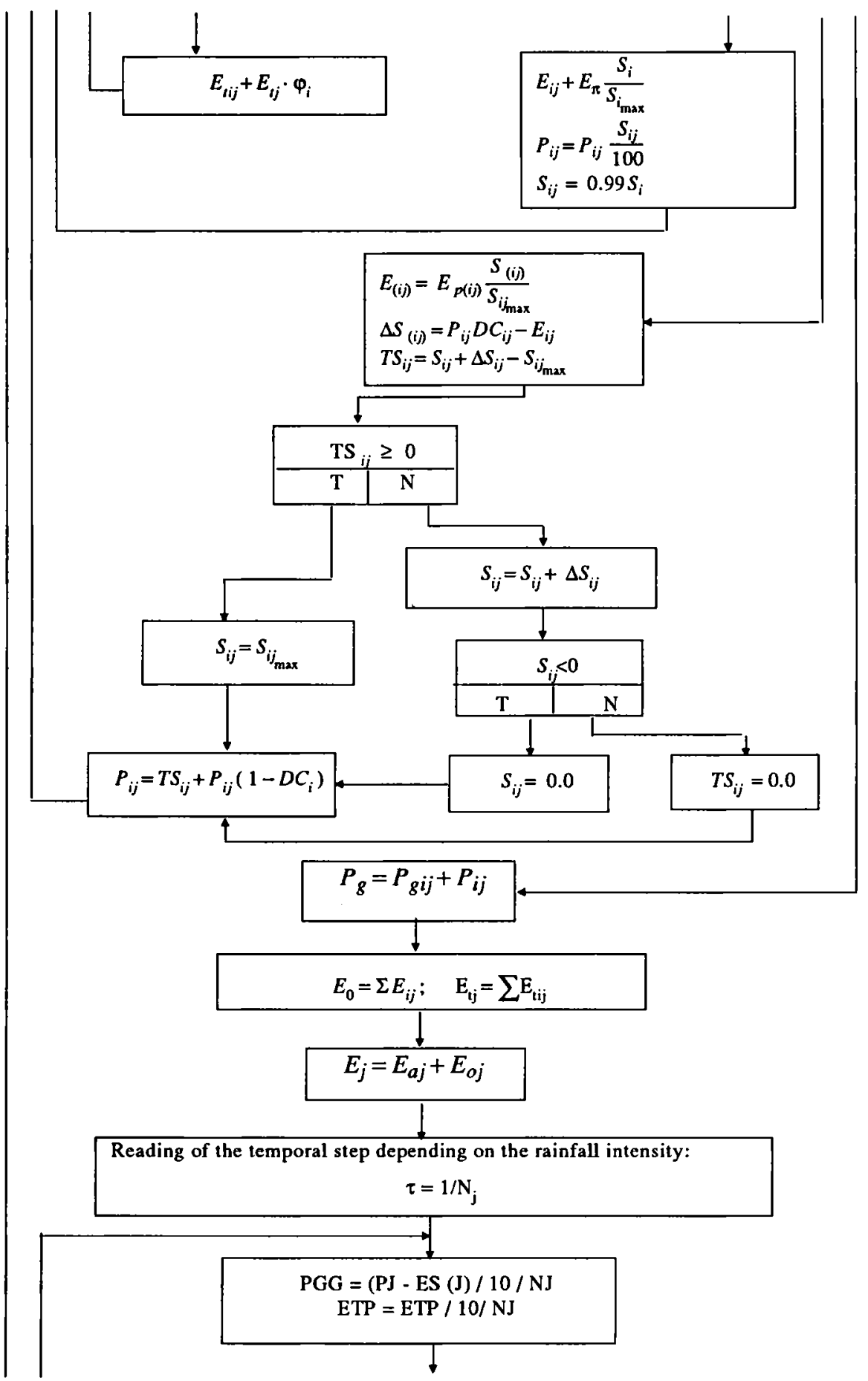




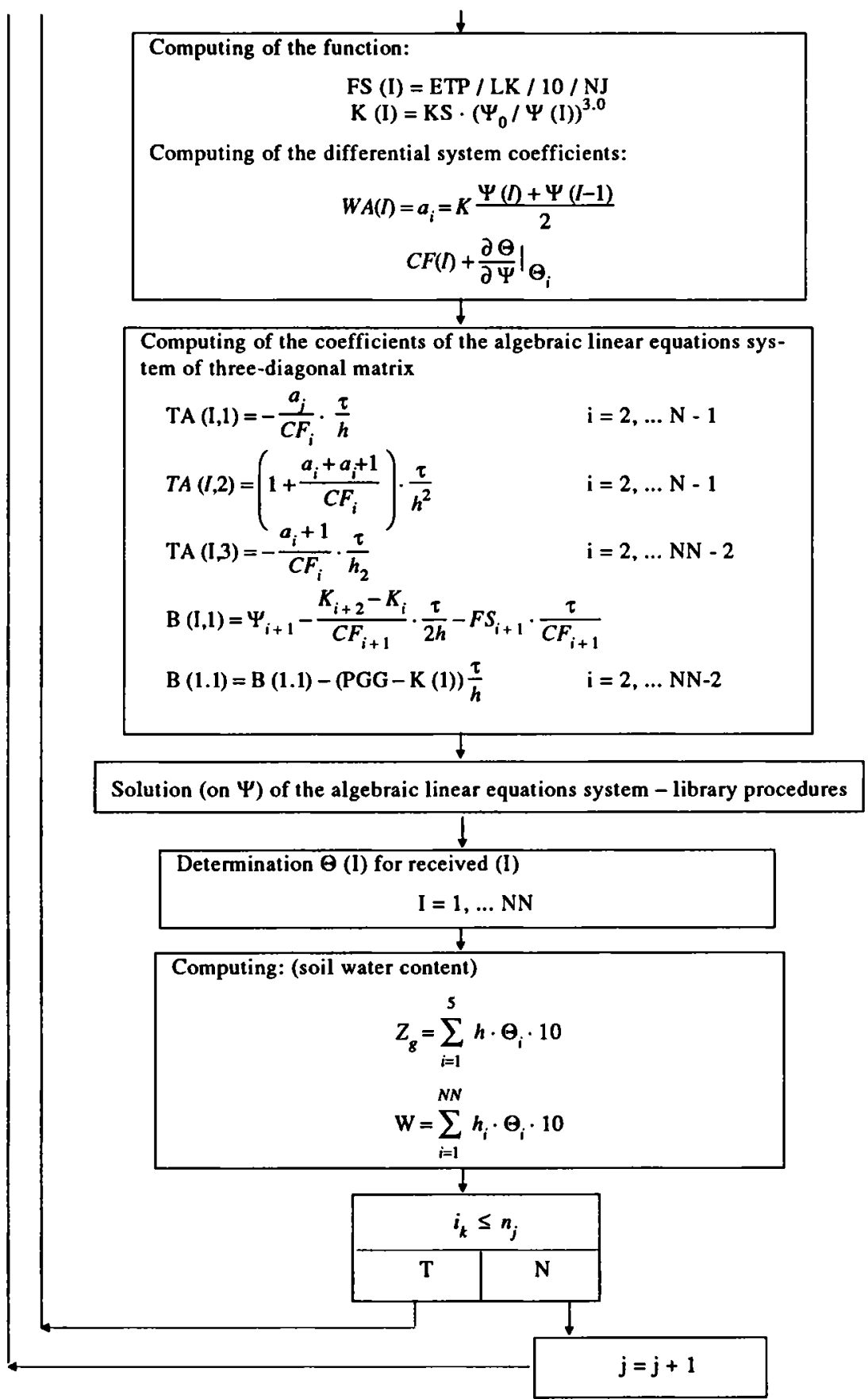

Fig. 1. Algorithm of the plant-soil column model (one-dimensional version, time step $1 \mathrm{~h}$ ) 


\section{EVAPOTRANSPIRATION}

Estimation of the evapotranspiration has been based on the algorithm elaborated by Jaworski (1978) being a physical adaptation of Montheith-van Bavel concept. It allows transformation of the energy influx from atmosphere to the plant canopy and soil into real evaporation from the ground as well as transpiration. The model is composed of 3 subsystems encompassing the atmospheric, plant and soil environments.

\section{SOIL MOISTURE CHANGES}

Infiltration has been treated as one-dimensional flow through a soil profile extending from the ground surface to the groundwater table. Within the active growth period, infiltration has been solved with Richards equation (SHE, 1987):

$$
\frac{\delta \theta}{\delta t}=\frac{\delta}{\delta z}\left(D(\theta) \frac{\delta \theta}{\delta z}\right)-\frac{\delta}{\delta z} K(\theta)-S
$$

where $t$ - time variable, $z$ - vertical ordinate, $\theta$ - soil moisture, $D(\theta)-$ soil water diffusion, $K(\theta)$ - hydraulic conductivity, $S$ - root water uptake.Two relationships are needed to be known in order to solve the equation: soil water diffusion $D(\Theta)$ and the hydraulic conductivity $K(\Theta)$. The equation has been approximated by the differentiation scheme.

Root water uptake $S$ has been determined by very simple approach

$$
S=\alpha(\Psi) \frac{E_{t p}}{l_{k}}
$$

where $E_{\mathrm{tp}}$ - potential evapotranspiration defined as the difference between potential $E_{\mathrm{p}}$ and soil evaporation $E_{\mathrm{s}} ; l_{\mathrm{k}}$ - depth of the root zone, $\alpha(\Psi)-$ reduction coefficient dependent upon plant access to soil water.

\section{MODEL VERIFICATION}

The one-dimensional mode with one hour time steps has been tested in the experimental field of the Warsaw University Mazovian Geographical Observatory in Murzynowo near Plock. Verification has been conducted in the soil profile under the spruce forest (column I) and under the fallow (column II). The model was tested in two steps: first on the basis of rainfall measurements under the trees crowns (col.I) and the second by comparing measured and computed soil moisture profiles (col. I and II). Results of the verification have been found to be fairly satisfactory (Soczyniska, Gutry-Korycka, Desperat, 1991). 


\section{NUMERICAL SIMULATION OF CHANGES OF THE HYDROLOGICAL PROCESSES}

Concept of the physically-based model is universal. Its universality results from the possibility of using it in various environmental conditions, by describing all processes with the same equations of mathematical-physics but different parameters (Soczyńska, 1986). Research is based on the assumtion that each change in the physical environment of the soil column or/and change of the water control in it should result in different course of hydrological processes in vertical water movement. The model has been used to predict the hydrological processes behaviour under the influence of the simulated various kinds of human activity within the unit basin.

The task of numerical simulation was to introduce changes in the physical environment of the unit basin area (e.g. reforestation, deforestation), changes of crop cultures, or changes in water distribution connected with raising or lowering of the underground water table or with application of irrigation in quantities necessary for growing the cultivated crops. Two types of simulations have been carried out: the first one for estimation of the physical changes influence on the dynamic system reaction and the second for investigation of its behaviour under the influence of changes in water cycle control. All numerical experiments have been performed within the active growth period.

\section{SIMULATION OF THE PLANT CANOPY CHANGES}

All simulations confirmed importance of the plant canopy in forming the rainfall feeding, evapotranspiration as well as changes of the soil moisture storage.

Relations between the potential evaporation and evapotranspiration are mainly governed by the meteorological element, but the plant canopy modifies them considerably. To a lesser extent it decides about the soil moisture storage and intensity of its changes. The biggest variability of the soil moisture profile, in spite of the ground kind, was observed down to $50 \mathrm{~cm}$ depth and it was closely connected with kind of the plant cover.

The biggest change of moistening of the whole soil profile (to $1,5 \mathrm{~m}$ ) was caused by full deforestation. It has an influence on behaviour of all analysed processes. The net rainfall increased by ca $12 \%$, the evaporation decreased aproximately twice and evapotranspiration has been displaced by the evaporation from the ground. Deforestation has also influenced the moistening of the soil profile. Down to $40 \mathrm{~cm}$ depth more than twofold increase of soil moisture was observed; below to $80 \mathrm{~cm}$ - the soil moisture decreases significantly. More below again a gradual increase of water content began to reach the saturation state at the groundwater level.

Under deforestation, the total water content in the soil profile (down do 1.5 $\mathrm{m}$ ) increased ca by $50 \%$ in relation to the tested profile. 
Effects of reforestation based on gradual increasing of the forest density and change in its structure by enlarging the interception area were simulated in a few variants in column $I$.

Condensation of the biosphere influenced increasing the interception storage and decreasing the net rainfall by ca $30 \%$. As a result of interception and increasing evapotranspiration the loss of soil content was approximately $30 \%$. Reforestation of the fallow land (column II) influenced decreasing more than $20 \%$ of soil moisture in the layer down to $70 \mathrm{~cm}$ depth (the maximum root zone). The potential evaporation and evapotranspiration increased very much and so did their quantity relations and the daily rythm.

\section{CHANGES OF THE CROP CULTURES}

Agrocenosis only slightly influences the soil moisture profile down to 80-90 $\mathrm{cm}$ of depth. Influence of the root crops and meadows is very similar. During the phase of the milky maturity, crops distinguish themselves among cultures and their influence on soil moisture decreasing is the biggest.

Locating the meadow on a post-forest and post-fallow land influenced only slightly the net rainfall and soil water storage. More changes were observed in potential evaporation and evapotransipration from particular cultures and ground layers.

It may be said that with the increasing interception area, the transpiration ability of thr phytocenosis increases more than that of the agrocenosis.

As a result of the interception increase, decreases infiltration and rising forest transpiration intesifies the water uptake by roots.

\section{CHANGES DUE TO WATER DISTRIBUTION}

The model tested in column I (forest) has also been used to simulate the change in hydrological processes in the soil profile under the influence of the raising and lowering of the underground water table. The raising of the ground water level up to $100 \mathrm{~cm}$ causes the solution destability of the equations describing the soil water contents changes. The results obtained cannot be treated as reliable.

The lowering of the ground water level from $1.5 \mathrm{~m}$ to $2.0 \mathrm{~m}$ caused (after 72 hours) decreasing about $22 \%$ of moisture storage in upper soil layers. Between $50 \mathrm{~cm}$ to $160 \mathrm{~cm}$ of depth soil moisture stays on the level of $12 \%-14 \%$. Below $160 \mathrm{~cm}$ the moisture gradient is very high $(\Delta \Theta=0.15$ to 0.30$)$ up to reaching the saturation state.

In column II (fallow) the model has been used to simulate the artificial irrigation of the soil profile covered with various cultures (meadow, rye, potatoes).

As it results from the numerical simulation, rye required watering during the whole period of 72 hours; the soil under this culture has not achieved the water field capacity; all moisture has been used on interception and evapotranspiration. 
The soil under meadow has already reached the water field capacity within second hour; during the last 70 hours there was observed a surplus of soil moisture. The soil under potatoes has reached the water field capacity within 5 hours.

The net irrigation intensities considered as the additional vertical soil watering depend upon the kind of the crop cultures, which affects the volume of interception, evapotranspiration as well as changes in soil water content.

\section{CONCLUSIONS}

Summing up the results achieved from the numerical simulations one may assess the tendency of changes of the hydrological processes due to the basin area utilisation. The attempt at anthropogenic simulations in the basin (steering of the ground water table) as well as irrigation of various cultures allows us to find the optimal direction of human activity for planning the purposeful catchment management.

The investigations were performed in the years 1986-1990 within the framework of the Central Programme of Fundamental Research No. 03.09 "Methods of Analysis and Use of Water Resources". A full description of the model algorithm and its application may be found in the paper by Soczyńska, Gutry-Korycka and Desperat (1991).

\section{REFERENCES}

Fle ming G., 1975, Computer simulation techniques in hydrology. Elsevier, New York, Oxford, Amsterdam.

Gutry-Kory cka M., S oczy ńska U., 1986, "Model of interception of plant canopy", Proceedings of International Conference on Hydrological Processes in the Catchment, Cracow, May 8-11.

Jaworski J., 1978, "A mathematical model of actual evapotranspiration), Journal of Hydrological Sciences No. 3-4, Warszawa

SHE, 1987, Système Hydrologique Européen.. A short description, Danish Hydraulic Institute, Horsholm.

S oczyńska U., 1986, "Prediction of anthropogenic changes in the hydrological cycle", Miscellanea Geographica, Uniwersytet Warszawski, Warszawa.

Soczyńska U., Gutry-Korycka M., Desperat T., 1991, "Processes of vertical moisture transport in quasi-homogeneaus unit field" in: Modelling of hydrological cycle. Atmosphere-Biosphere-Pedosphere, Uniwersytet Warszawski, Warszawa. 